Itinéraires Itinéraires

Littérature, textes, cultures

\title{
Mots, textes et images : tours de passe-passe dans les artist web projects d'Allen Ruppersberg et d'Iñaki Bonillas
}

Words, Texts and Images: Sleights of Hand in the Artist Web Projects by Allen Ruppersberg and Iñaki Bonillas

\section{Constance Moréteau}

\section{OpenEdition}

Journals

Édition électronique

URL : http://journals.openedition.org/itineraires/3923

DOI : 10.4000/itineraires.3923

ISSN : 2427-920X

\section{Éditeur}

Pléiade

\section{Référence électronique}

Constance Moréteau, « Mots, textes et images : tours de passe-passe dans les artist web projects d'Allen Ruppersberg et d'Iñaki Bonillas », Itinéraires [En ligne], 2017-3 | 2018, mis en ligne le 15 juin 2018, consulté le 01 mai 2019. URL : http://journals.openedition.org/itineraires/3923 ; DOI : 10.4000/ itineraires.3923

Ce document a été généré automatiquement le 1 mai 2019.

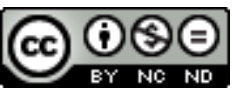

Itinéraires est mis à disposition selon les termes de la licence Creative Commons Attribution - Pas d'Utilisation Commerciale - Pas de Modification 4.0 International. 


\title{
Mots, textes et images : tours de passe-passe dans les artist web projects d'Allen Ruppersberg et d'Iñaki Bonillas
}

\author{
Words, Texts and Images: Sleights of Hand in the Artist Web Projects by Allen \\ Ruppersberg and Iñaki Bonillas
}

Constance Moréteau

Dans le contexte actuel d'une mise en ligne exponentielle d'archives et de collections, qui fonde en bonne partie les humanités numériques, il est intéressant de prendre en compte le positionnement d'artistes offrant une visibilité étendue à certaines de leurs sources visuelles et littéraires. Numérisées et codées, celles-ci intègrent des œuvres accessibles en ligne. Elles doivent être placées en vis-à-vis d'une véritable « obsession » (Roelstrate 2009) de nombreux artistes pour les archives, la mémoire, et plus généralement pour le passé qui, après avoir émergé dans les années 1960, occupe une place de premier plan dans l'art contemporain depuis une décennie environ. De nombreuses expressions viennent qualifier ce phénomène, témoignant ainsi de sa prégnance : «Archive Fever » traduit à partir du « Mal d'archive » de Jacques Derrida (1995), « Archival Impulse» (Foster 2004), ou encore "Artists as Historians» (Godfrey 2007), pour n'en citer que quelques-unes. Mark Godfrey, qui souligne la place souvent centrale de la photographie dans ces pratiques conceptuelles, fait aussi la distinction entre un intérêt pour l'opacité et le mystère de documents historiques revisités et l'exploration du passé (Godfrey 2007 : 142). Les synthèses sur le rapport à l'histoire des artistes contemporains se sont multipliées au cours de la dernière décennie, aussi nous préférons ne pas réitérer cet exercice et nous renvoyons aux travaux de Mark Godfrey déjà cités, de Dieter Roelstraete, de Vivian Rehberg (2010), d'Okwui Enwesor (2008) et à l'ouvrage collectif L'avenir du passé. Art contemporain et politiques de l'archive, paru en 2016 (Zapperi 2016). Dans l'introduction de ce dernier, Giovanna Zapperi montre que l'intérêt pour ces pratiques s'explique par leur localisation « dans un espace liminaire entre la représentation et la production du passé, 
entre le réel et la fiction, le public et le privé, l'objectivité et la subjectivité » (Zapperi 2016: 7). En revanche, une attention moins conséquente a été accordée aux œuvres numériques traitant de cette question, sans doute en raison de la marginalité à laquelle elles sont encore reléguées dans l'histoire de l'art. Or celles abordant la problématique de la mémoire ou de l'archive sont par exemple nombreuses au sein du vaste programme Artists Web Project lancé en 1995 par la Dia Foundation ${ }^{1}$, pionnière dans le soutien des œuvres numériques ${ }^{2}$. On peut ainsi relever les exemples suivants: Words and Images d'Iñaki Bonillas (2014), John Brown Song! de Laylah Ali (2013), Vertiginous Mapping de Rosa Barba (2008), The New Five Foot Shelf, d'Allen Ruppersberg (2004), Annotations de Glenn Ligon (2003), Phantom Tales de Feng Mengbo (2001) et Wake de Gary Simmons (2000). L'étude de ces web projects vient enrichir la réflexion sur les différentes dualités, énumérées par Giovanna Zapperi, dans la mesure où ce sont des œuvres que seule l'activation du spectateur peut pleinement déployer. Construite à partir d'interfaces logicielles et visuelles, une œuvre numérique joue de sa profondeur; et son épaisseur émane d'abord de cette réalité technique. Pour reprendre Jean-Paul Fourmentraux «la double fonction de l'interface est de fournir une représentation perceptible de la profondeur de l'œuvre conçue par l'auteur, dans l'objectif de former, en second lieu, un théâtre d'opérations pour ses utilisateurs » (Fourmentraux 2008). Pour commencer par la première particularité qu'il énonce, la récurrence de cette thématique de l'archive pour ce médium peut s'expliquer par la possible correspondance entre les strates d'une œuvre numérique et celles qui constituent la mémoire et/ou l'archive. Pour les web projects d'Allen Ruppersberg ${ }^{3}$ et d'Iñaki Bonillas ${ }^{4}$, par exemple, cette épaisseur se révèle dans ses multiples entrées au travers de l'indexation qui les ordonne. Cette indexation témoigne de la nature de la relation entre les archives réelles et leurs doubles numériques. Comme le dit, en juin 2014, Iñaki Bonillas dans un entretien public avec Manuel Cirauqui, curator à la Dia Fondation, expliquant son choix de créer au sein de l'environnement sociotechnique d'Internet : "J'ai trouvé cela séduisant car Internet est déjà une réplique du monde. Parce que le cadre était en soi équivalent à un jeu de miroirs, j'ai pensé qu'il pourrait être profond de produire un autre double en utilisant les mots ${ }^{5}$.» Dans le cas d'Iñaki Bonillas, les archives sont composées de la très importante œuvre photographique de son grand-père dont il assume ne pas connaître toute l'ampleur et tous les enjeux (Ibid .). Au contraire, pour Allen Ruppersberg, celles-ci ont été rassemblées par lui-même et la problématique du passé tient d'abord à l'immortalisation photographique de ce fonds tel qu'installé à New York avant que l'artiste déménage et dont il connaît tous les recoins. C'est aussi sa propre mémoire de son lieu de travail qu'il porte et fixe. On peut se demander si la déambulation dans une forme de reconstitution virtuelle de celui-ci n'est pas aussi pensée pour son propre usage personnel.

Pour les deux artistes, l'indexation passe en grande partie par un rapport entre le texte et l'image et elle peut être étudiée au travers d'un prisme littéraire. Word and Image est défini comme de la poésie concrète par Manuel Cirauqui, et on pourrait parler de collage littéraire dans le cas d'Allen Ruppersberg. Au centre de son dispositif, ce dernier place un de ses livres d'artiste qui, portant le même titre que le web project, est essentiellement constitué de citations et de textes personnels. Pour les deux artistes, l'apport linguistique occupe une fenêtre sur l'écran tandis que les images en occupent une autre et ces deux entités peuvent être activées à différents rythmes mais elles ne sont pleinement signifiantes que lorsqu'elles sont mises en relation. Toutefois, il importe de considérer d'abord cette indexation sous son angle technique, en ce qu'elle peut nous renseigner sur 
la place conférée au degré d'autonomie de l'artiste auteur et de l'utilisateur. Friedrich Kittler, considéré comme le père de l'archéologie des médias, tient à marquer une distinction entre le simple utilisateur - que nous identifions autant comme l'artiste que l'utilisateur - qui utiliserait les logiciels comme des outils maîtrisés à un degré superficiel, et le véritable auteur qui disposerait d'une meilleure maîtrise de la machine (Kittler $2015^{6}$ ). Ainsi Iñaki Bonillas est assisté par l'artiste mexicain Iván Abreu qui est également programmateur informatique du design studio Taller de Comunicación Gráfica. En revanche, aucun programmateur n'est indiqué pour The New Five Foot Shelf où l'indexation reste à un niveau technique superficiel. Pour cette œuvre, il s'agit simplement de combiner les pages du livre et les images de l'atelier de l'artiste en un nombre presque infini de possibilités comme si, finalement, toutes les composantes de l'atelier avaient pu être intégrées dans la conception du livre ; on comprend alors qu'il n'y a pas eu de nécessité d'encodage contrairement à l'œuvre d'Iñaki Bonillas, où toutes les images ne peuvent être associées, et plus encore définies, par le texte. L'accès aux photos est suspendu à leur encodage ; or celui-ci se base d'abord sur une approche linguistique et parfois poétique qui condamne certaines photographies à ne jamais s'afficher à la surface de l'écran en dépit de la simplicité du dispositif technique. Pour les œuvres du programme Dia Artist Web Projects, il est la plupart du temps pertinent de parler de software-based works ${ }^{7}$ - une dénomination très approximative que l'on pourrait traduire par « œuvres logicielles »-, ce qui traduit un certain détachement de la part des artistes vis-à-vis d'une approche réflexive portée sur les nouvelles technologies. On remarque d'ailleurs la simplicité de l'usage des œuvres au moins à un niveau technique. En revanche, dans les deux œuvres le degré d'accès à la totalité de leurs composantes est suspendu à des conditionnements culturels, et ce sont ces conditionnements qu'il s'agira ici d'étudier. Indexer et/ou interroger l'index mis en place revient souvent à révéler les manques de notre définition du monde que même une mise en accès généreuse ne comble pas 8 .

\section{Words and Photos, Iñaki Bonillas, 2014 : une indexation mise en interrogation}

3 Words and Photos, mis en ligne en 2014, se construit à partir de l'impressionnant fonds d'archives du grand-père maternel de l'artiste décédé en 2000, José Rodríguez Plaza, auteur de l'ensemble des quelques 3800 photographies qui le composent. Depuis 2003, l'ensemble de ces documents est réapproprié sous le titre générique J. R. Plaza Archive. Ses déclinaisons sont multiples et prennent part à une réflexion de grande ampleur portant principalement sur la photographie; la mémoire est une thématique secondaire tout comme celle de l'identité. L'association entre photographies et mots est résumée de manière lapidaire dans le texte de présentation du projet, consultable sur le portail des web projects de la Dia Foundation :

Bonillas numérise les archives, tout en créant simultanément un index de mots associés qui « reflètent " [mirrors] la base d'images. La relation entre ces niveaux se déploie au fur et à mesure que le visiteur explore les possibilités d'association entre le texte et l'image. Débutant avec un ensemble de 400 images, l'œuvre va progressivement se développer jusqu'à ce que toute l'archive J. R. Plaza soit cartographiée ${ }^{9}$.

À notre connaissance, deux spécificités distinguent cette œuvre par rapport aux autres déclinaisons du projet: l'ensemble des photographies de l'archive est inclus dans une même œuvre et la participation des spectateurs est de nature collaborative, du moins 
dans un premier temps. Avant que l'index soit mis à la disposition de tous les utilisateurs, l'artiste invite des poètes, des écrivains et l'équipe de la Dia Foundation à collaborer à l'indexation du projet, qui inscrit l'intersubjectivité au cœur de celui-ci, comme le souligne bien l'artiste :

Dans une photographie, par exemple, une femme pleure; d'un coup de tête, il aurait été prévisible de la taguer avec un mot qui décrit son abandon, mais cela aurait pu être incorrect. Cette personne pouvait bien pleurer non de tristesse mais de joie. Une pluralité de sens existe et peut dériver d'une archive personnelle de mots, d'une mémoire, ou d'une histoire personnelle unique qui est différente de la mienne ${ }^{10}$.

Chaque occurrence de l'index n'excède pas un mot, obligeant l'utilisateur à définir sa vision de chaque photographie avec une concision extrême. La participation de poètes et d'écrivains accentue l'amarrage d'univers très personnels à la constitution de ce projet. Le choix d'un seul mot à chaque fois par chaque participant est donc empli d'une forme de dramatisme, qui fait osciller la réception par des utilisateurs, que nous nommerons "postérieurs ${ }^{11}$ ", entre interrogation de ces définitions posthumes et interrogation de l'intention de l'auteur même des photographies. Mais avant d'en arriver à ce niveau, l'utilisateur postérieur doit interroger la base elle-même. À chaque nouvelle connexion, l'utilisateur fait face à une page vierge pour une large part : en haut à gauche, une petite interface invite à entrer un mot "enter a word ». Pas même une fenêtre délimitée ne suggère un espace réservé pour la photographie ; seul le vide l'indique en creux. Celle-ci ne se profilera qu'au moment de l'apparition d'une série de photographies sélectionnée par le logiciel. Au tout début de la consultation de l'œuvre, seules deux lignes de mots apparaissent dans la partie inférieure de l'écran, sans qu'on sache, lors d'un premier usage, ce à quoi ils se réfèrent. Il semble ainsi, au premier abord, que la base est presque un lieu universel où tout mot peut potentiellement relier la mémoire de l'auteur des photographies au monde plus vaste. Cela peut aussi apparaitre comme une manière de ne pas censurer chaque utilisateur postérieur, libre d'inscrire ce qui lui traverse alors l'esprit avant d'être contaminé par un univers formé de multiples voix comme si la base offrait presque deux fonctions: celle d'explorer la vision de José Rodríguez Plaza et celle d'assumer l'incomplétude évidente de l'indexation. Pour revenir à l'index, celui-ci se déroule sur deux lignes disposées à l'horizontal. Ces lignes vont être augmentées par les termes nouvellement entrés par l'utilisateur postérieur, tels ceux que nous proposons en écrivant cet essai et sur lesquels nous reviendrons ultérieurement. Les mots retenus avec succès sont remémorés dans la ligne supérieure de l'index. Certains mots sont refusés au sens où leurs inscriptions dans la fenêtre intitulée « enter a word » sont effacés sitôt leur saisie faite, ne générant donc aucune série de photographies et se trouvant néanmoins mémorisés dans la ligne inférieure de l'index. Ce sont des mots qui n'ont pas été sélectionnés au moment de la phase d'élaboration collective de l'œuvre mais, il faut le souligner, qui apparaissent néanmoins. Dans la ligne de ces mots refusés, un même mot peut être reproduit à plusieurs reprises sans interruption. Lors d'une consultation les mots refusés suivants s'offrent ainsi successivement: «impossibility, imposition, calculator, calculator, calculator, pickle, sex, sex, france, sex, sex, sex, sex, sex, drugs ". Très certainement, la répétition tient de l'étonnement et de l'insistance de l'utilisateur, qui ne comprend pas le mutisme de la case réservée aux images, ou bien de son amusement à créer une répétition dès lors que le fonctionnement de l'inventaire a été compris. Cette répétition peut être aussi assurée par une autre personne. 
6 Un autre mode d'exploration de l'archive est aussi possible, en croisant l'image et le mot retenu. Par exemple, l'exploration du web project peut se poursuivre en cliquant sur l'un des mots qualifiant une photographie qui lui-même peut être associé à d'autres photographies indexées. Le nombre de mots indexant une photographie peut être très important. Ainsi en va-t-il de l'autoportrait en pied et en noir et blanc de José Rodríguez Plaza pour lequel celui-ci pose quasiment de profil dans un paysage désertique, lequel est indexé par 183 mots différents. L'un d'entre eux est « nervous » qui, lui, est apposé à cinq images. D'autres suggèrent de nombreuses contradictions qui marquent l'intersubjectivité innervant son interprétation ou, plus simplement, sa description: « open", « white», « impending", «picturesque », « lips», etc. Jamais l'utilisateur ne pourra disposer d'une vision simultanée de toutes les archives photographiques. Plutôt que d'épuiser la connaissance de l'archive, l'utilisateur doit s'attendre à voir ressurgir une même photographie indexée avec plusieurs mots.

7 Pour faire vaguement écho à la thématique du numéro d'Itinéraires, nous proposons le mot «writing" et la nature expérimentale du résultat tient alors des indexations surprenantes qui peuvent en découler: dix photographies, toujours les mêmes, sont sélectionnées selon un ordre aléatoire d'une connexion à l'autre, lorsque ce même mot est de nouveau entré. Le mot a donc déjà été utilisé pour qualifier un certain nombre de photographies. Le projet étant maintenant clos dans son élaboration, nous ne pouvons encoder de nouvelles images. Au gré du rythme de l'utilisateur, les photographies se succèdent l'une après l'autre. Il n'est pas toujours évident de comprendre le sens de certaines images telles que considérées dans le contexte du mot référent. Pour "writing ", la première photographie, par exemple, donne à voir un groupe d'individus, nous faisant sans doute remonter dans les années 1930 et pour lequel plus d'une centaine de mots ont été associés; notons d'ailleurs que dans ce projet, aucune image n'est légendée. Il semble que ce soit les quelques lettres visibles sur la façade tronquée par le cadrage «BLICK » qui justifient cette catégorisation. Aucune connaissance personnelle des photographies ne vient interférer avec l'indexation. De même, dans l'arrière-plan d'une photo de famille, des textes imprimés illisibles sont encadrés. Plusieurs autres photographies sont aussi compliquées à comprendre dans le contexte du mot saisi. Puis on découvre une autre photographie capturant une petite bibliothèque supportant des volumes encyclopédiques. L'écriture comme processus est clairement identifiée dans la troisième photographie, un autoportrait de José Rodríguez Plaza écrivant dans un train. Enfin, pour d'autres photographies, le lien semble s'expliquer par la présence d'une table - une table dressée pour un repas - support traditionnel de l'écriture. Les consultations du site se font souvent dans la perplexité, que ce soit en prenant connaissance de certains mots associés aux images ou, au contraire, de l'absence de certains qui semblaient convenir sans hésitation, du moins nous concernant. Dans un entretien livré à Franck Espósito, en novembre 2014, l'artiste avoue d'ailleurs son étonnement face à certaines indexations manquantes :

Dans d'autres, un mot aussi évident que «narcissisme " pour un autoportrait n'a pas été tagué initialement. Mon grand-père apparaît comme un homme vaniteux dans beaucoup de ces photos; il se pensait comme un acteur d'Hollywood et s'appropriait la personnalité de John Wayne en cowboy avec un pistolet pour un grand nombre. Mais personne dans le projet n'a indexé ces photos avec "narcissisme». Ce mot existe sur le site, cependant, parce qu'au moins un utilisateur l'a cherché ${ }^{12}$. Tous ceux absents mais cherchés en tant que tags, sont automatiquement ajoutés à une liste située en bas de l'écran. Plus je vois ces mots et plus je me demande pourquoi la personne les a choisis. Qu'est-ce qu'ils voient que je 
ne vois pas? Parfois les éléments que vous avez en face de vos yeux vous sont impossibles à voir. C'est le résultat le plus incroyable de ce projet : nos limitations. Comme Borges le disait, personne ne peut jamais finir de décrire une image puisque pour celle-ci il y a un océan de mots ${ }^{13}$.

Cette relation complexe à l'index nous ramène à des lectures sémiologiques de l'image et de la photographie introduites dans l'histoire de l'art et plus spécifiquement dans l'histoire de la photographie à partir des années 1970 et ce jusque dans les années 1980. Le titre même de l'œuvre Words and Photos semble s'y référer, faisant écho aux lectures indicielles et/ou intentionnellement significatives de la photographie ${ }^{14}$, qui s'élaborent aux États-Unis et en Europe. Il s'agit alors de soutenir la reconnaissance de la photographie comme un art à part entière ou de l'insérer dans une méthode d'interprétation linguistique de l'art, dialoguant avec le poststructuralisme alors en vogue. Selon les théoriciens s'étant de la sorte penchés sur la photographie, l'index ne revêt pas toujours le même sens, qu'il s'agisse pour la théoricienne américaine Rosalind Krauss, dans son essai « Notes on the Index: Seventies Art in America ", publié en 1977, d'un signe par connexion physique, en référence au logicien américain Charles Peirce ou, pour le Belge Henri Van Lier, d'une définition de l'index fondée essentiellement sur sa distinction du signe dans son article "La rhétorique des index" paru en 1982. "La rhétorique des index » amène à une lecture plus subtile de la photographie, appréhendée dans ses ambiguïtés. Selon Henri Van Lier, "l'index indique au sens fort», il est « intentionnel et conventionnel» (Van Lier $1982: 14$ ). Toutefois, pour Words and Images, lorsque l'indexation se produit au prisme du détail, cette adhésion à la réalité est-elle davantage le fait de José Rodríguez Plaza ou des collaborateurs d'Iñaki Bonillas? L'indexation du réel engagée dans et par la photographie au moment de sa conception, par José Rodríguez Plaza, bute sur celle encadrée par son petit-fils. Or le télescopage entre les deux ne résulte pas uniquement de l'opération menée par Iñaki Bonillas, que l'on ne peut interpréter sous la forme d'un parasitage. Le poète et professeur de littérature mexicain Luis Felipe Fabre décrit ainsi le phénomène dans un essai développé sous une forme épistolaire: "Je ne peux m'empêcher d'imaginer que les images dans les photographies de votre grand-père disparaissent au moment où elles deviennent des images photographiées ${ }^{15}$ » (Fabre 2006: 56). L'intentionnalité à l'origine de ces photographies rend presque autant étranger à celles-ci l'artiste que ses collaborateurs. $\mathrm{Ne}$ pas supprimer les mots non inclus dans l'index, c'est aussi reconnaître l'égalité presque totale du statut de l'artiste avec celui des utilisateurs de l'œuvre. Les photographies tendent ainsi à être considérées comme des images trouvées. Le reconnaître oblige aussi à admettre la grande relativité qui entoure la notion d'index dans Words and Photos : quelle part d'intention est en effet donnée originellement (au moment de la prise photographique) à la capture photographique d'une table ou d'une femme à la pose langoureuse? L'image est souvent saisie par des détails qui, pour celle-ci, ne seront pas les mêmes d'une indexation à l'autre. Van Lier, qualifie de "rhétorique des index " l'articulation des éléments indexés en ce qu'elle "concerne des ensembles vastes et flottants; elle implique toujours un certain bruit» (1982: 15). Ce bruit est produit notamment par cette ligne de mots sans image associée, parce qu'on ne sait quelle place leur donner et existant malgré tout. S'il y a une forme de parasitage, il intervient à ce niveau et il a le potentiel d'enrichir la lecture des photographies. Van Lier préfère cette définition à celle de syntaxe qui « est une pratique rigide s'appliquant à des éléments également très définis, ce qui n'existe nullement dans la photo " (Ibid.). La distinction que postule Van Lier entre l'indice et le signe, que Peirce n'a pas établie, est intéressante à 
considérer pour la présente étude de cas puisqu'il souligne "qu'il y a des photos très indexées, d'autres peu, d'autres pas du tout", préférant alors se saisir de la notion d' imprégnant, soit «la cause d'un effet, manifestée à travers cet effet» (Ibid.). Dans des photographies, si l'on suit Van Lier, il subsisterait des « plages sans imprégnant repérable ${ }^{16}$ " (Ibid. : 16). Un certain nombre de photographies de Words and Photos sont d'ailleurs susceptibles d'échapper à toute indexation poussée si elles ne réapparaissent pas au hasard des interrogations par l'image et/ou le mot. L'indexation apparemment infinie de l'archive photographique pourrait ainsi apparaitre comme une tentative vaine de définir les différentes portions de chaque photographie. Tout comme la liste des mots suspendus pourrait suggérer ce qui ne pourra jamais être vu ou ce qui n'aura pas pu accéder à une mémoire collective au moins si on choisit de l'approcher par un biais linguistique. Dans le même temps, ce qui semble parfois le moins contestable, ce sont les effets verbalisés produits par la photographie si toutefois la subjectivité de leur énonciation si minimaliste est bien prise en compte et acceptée.

\section{The New Five Foot Shelf, Allen Ruppersberg, 2004 : appuyer sur l'interrupteur on/off}

The New Five Foot Shelf, mis en ligne en 2004, clôture un projet portant le même titre, débuté en 2001 ; une création littéraire difficilement diffusable et associée à un atelier qui n'existe plus, occupé par l'artiste au 611 Broadway à New York entre 1986 et 2001. La publication déjà brièvement évoquée consiste en une réinterprétation de The Five- Foot Shelf, éditée par un doyen de l'université d'Harvard, Charles W. Eliot. Cette somme encyclopédique, publiée en 1910 chez Collier and Son, compile en 51 volumes les textes considérés comme les plus incontournables de la culture occidentale. De celle-ci, dans The New Five Foot Shelf, ne subsiste que l'introduction. Pour Charles W. Eliot, son ouvrage serait une bibliothèque plutôt qu'une encyclopédie, une définition qui, par sa portée physique que révèle le titre - soit le métrage de l'étagère la supportant - aurait pour mérite de promettre l'appropriation pleine et entière de ce savoir par le lecteur. Dans une nouvelle version, signalée par l'ajout du New, Allen Ruppersberg optimise l'idéal d'efficacité, que ne cesse de vanter le doyen, en commençant par la mise en page de sa publication. Les cinq chapitres qui le composent se développent sur quelques lignes et sont ainsi superposés les uns aux autres sur chaque page, séparés par un espace et une typographie différenciée : "Honey, I rearranged the collection », "When in Doubt Go to the Movies (notes). Chapter One. 611 Broadway », « Once Upon a Time When Books were Famous (works) », «The Three Marcels » et « The Master of the Familiar (private) ». Dans l'installation réalisée en 2001, les chapitres progressent sur un mode proche d'un défilement cinématographique, de volume en volume, 48 au total. Le dernier volume est réservé à un index photographique de l'atelier new-yorkais. En 2003, une très épaisse version papier rassemble le tout en un seul volume. Publiée en 1000 exemplaires par les éditions Micheline Szwajcer et Michèle Didier, elle ne peut être commercialisée comme l'indique la mention Not for Sale du colophon. En effet, nombre d'écrits compulsés dans cette œuvre sont en réalité des extraits des lectures de l'artiste, non exemptes de droit d'auteur. Pour autant, les extraits ne sont pas référencés dans la version de 2003 et l'ensemble n'est pas paginé. Il sera donc très difficile de citer l'ouvrage dont ces extraits, entremêlés avec des notes personnelles et un chapitre dévolu à cet atelier disparu, semblent démontrer une dépendance organique avec l'espace privé de l'artiste, rejouant 
ainsi la définition de l'encyclopédie par Charles W. Eliot. C'est ce que corrobore l'œuvre numérique au travers de la complémentarité entre le texte et l'image. L'indexation du texte dans l'espace numérique est d'ailleurs un enjeu complexe que relève Serge Bouchardon:

[...] le lecteur a l'impression que le volume exact du texte ne peut plus être évalué, que les «pages» web ne peuvent plus être touchées, feuilletées, que le texte disparaît dans un espace inconnu dès qu'un nouveau lieu a été activé. (Bouchardon 2008:9)

Avant de s'attarder sur la présentation du dispositif, il importe de revenir sur une partie du texte développée dans le premier chapitre, «Memoir/Novel/Index » :

L'atelier se transforme en un ensemble de livres.

L'ensemble de livres se transforme en une sculpture.

La sculpture se transforme en une installation.

L'installation se transforme en une pièce.

La pièce se transforme en photographies.

Les photographies se transforment en pages.

Les pages se transforment en livres.

Les livres se transforment en volumes.

Les volumes se transforment en un ensemble.

L'ensemble se transforme en une collection.

La collection se transforme en une bibliothèque.

La bibliothèque se transforme en posters.

Les posters se transforment en une sculpture.

La sculpture se transforme en architecture.

L'architecture se transforme en références.

Les références se transforment en index.

L'index se transforme en table des matières.

La table des matières se transforme en notes.

Les notes se transforment en légendes.

Les légendes se transforment en histoires.

Les histoires se transforment en roman.

Le roman se transforme en histoire.

L'histoire se transforme en objets.

Les objets se transforment en biographie.

La biographie se transforme en biographes.

Les biographes se transforment en un auteur.

L'auteur se transforme en un artiste.

L'artiste se transforme en un sujet.

Le sujet se transforme en un texte.

Le texte se transforme en un livre.

Le livre devient mon atelier ${ }^{17}$.

11 Il ne s'agit que d'un extrait; le texte s'étire le long d'une trame qui paraît presque infinie. Dans cette partie du livre présentée comme un mémoire, la relation de l'artiste à son environnement, tant intellectuel que matériel, témoigne d'une qualité littéraire diffuse. Cette dernière peut être résumée par l'interprétation que fait le photographe et théoricien canadien Jeff Wall de l'approche du livre et de la littérature chez un autre artiste conceptuel de la même génération qu'Allen Ruppersberg, Rodney Graham, lui aussi fasciné par le livre :

L'objet de Graham est une "chose» littéraire [Literary Thing] qui n'est pas réellement un livre mais qui doit se manifester comme un livre. La "chose » qui apparait ainsi est la pure manifestation d'une répétition-compulsion, pure dans le sens où elle est rendue visible non à travers l'acte d'une création littéraire mais par 
la ruine organisée de la littérature. La perte se produit non par l'écriture d'un livre mais par le fait de le relier ${ }^{18}$. (Wall $1988: 12$ )

Dans cette "chose littéraire" qu'Allen Ruppersberg ordonne d'abord comme une métamorphose perpétuelle, l'index devient une occurrence parmi d'autres et toute assignation pérenne est donc évacuée. Si l'on arrive à s'extraire de la circularité de "Memoir/Novel/Index", une question peut alors se poser : qu'indexe-t-on ? Comment indexe-t-on? Par la barre oblique qui divise les trois entités du titre, il semble que n'importe quelle relation les liant soit réversible. Dans l'œuvre numérique, cette action neutralisante de la reliure, telle que mentionnée par Jeff Wall, pourrait s'apparenter à la combinaison des trois fenêtres disposées sur un fond composé des vues photographiques de la bibliothèque new-yorkaise. La fenêtre du bas, qui occupe toute la partie droite de l'écran dans sa longueur, reproduit l'ensemble des cinquante volumes, visibles seulement à partir de leurs reliures de facture classique. La sélection d'un volume grâce au curseur permet l'activation de la fenêtre occupant toute la partie supérieure gauche de l'écran. Son format se confond avec celui des pages des volumes. Les pages du volume sélectionné peuvent défiler de gauche à droite et inversement. Quant à la fenêtre située dans la partie supérieure droite, elle fonctionne de manière plus indépendante puisque la consultation, une à une, des photographies de l'atelier ne dépend pas du choix d'un volume. Initialement, ces photographies composaient l'arrière-plan de l'installation de 2001 alors que, sur le devant, l'ensemble des volumes était sobrement disposé sur une planche posée sur des chevalets. Afin de restituer la tridimensionnalité de l'atelier, il est possible de faire défiler les images de la gauche vers la droite ou dans l'autre sens, mais aussi du haut vers le bas et inversement. La taille équivalente des pages du livre et des photographies crée un sentiment de proximité qui est nouveau si on compare le web project aux versions antérieures de l'œuvre. Le texte peut être associé à toutes les images si bien que la fonction indicielle des photographies dans l'installation est très relative. Le hors-cadre qui prévaut, et met en avant les interstices des rayonnages et des espaces de l'atelier, montre que l'ultra-indexation photographique de l'espace trahit le regard ironique porté par l'artiste sur toute tentative d'approche génétique de son œuvre ${ }^{19}$. Tout simplement, l'attention de celui qui cherche est souvent conditionnée : un petit détail vient appuyer ce qui est d'abord de l'ordre du constat, s'agissant d'un très discret interrupteur situé tout en haut à droite de l'écran. Dès qu'il est mis en position de marche, des extraits de films et de chansons sont diffusés. Or il est très rarement remarqué par les utilisateurs, comme le note l'artiste ${ }^{20}$. Pourtant la bande sonore vient ajouter la musique à la diversité des archives de l'artiste. Elle serait jugée digne d'attention par l'utilisateur de l'œuvre, si ce dernier avait en tête la vision de la bibliothèque par Friedrich Kittler plutôt que celle moins complète proposée par Michel Foucault. Cette dernière se limite en effet à la conservation des énoncés et sert encore largement aujourd'hui de modèle épistémologique alors que Kittler insiste sur la dimension multimédiale de la bibliothèque. Ainsi la mise en marche de l'interrupteur par le spectateur dépend de sa réceptivité à la complexité de la "chose littéraire» dont la totalité est difficile à embrasser. On serait tenté de parler d'ultra-indexation qui à ce point confinerait à l'absurdité. Ce petit détail de l'interrupteur montre bien plutôt qu'on ne se saisit que d'une partie d'une culture personnelle, parfois extra-artistique à l'origine, et à laquelle on indexe entièrement une œuvre d'artiste. 
Du bruit comme métaphore d'une indexation parasitaire chez Iñaki Bonillas à la bande sonore qui densifie presque par hasard l'objet exploré, l'expérience de l'œuvre se nourrit également de ce qui se situe à la marge. Pourtant, loin de signifier une hiérarchisation de l'univers aussi visuel, linguistique, littéraire, émotionnel que sonore, ces œuvres montrent d'abord ce qui échappe à celui qui cartographie. Pour Words and Images, cela tient avant tout à un filtre, autrement dit l'indexation incomplète des collaborateurs rassemblés par l'artiste dans un premier temps qui peut s'expliquer par une sorte d'autocensure, un imaginaire apposé à tous les objets d'étude et à un spectre très large d'intention et d'inattention, une dernière caractéristique que l'on retrouve aussi au cœur de la problématique de The New Five Foot Shelf. Dans le second cas, le livre d'artiste numérisé fournit en soi toutes les clés de compréhension de l'univers créatif de l'artiste. S'il n'est pas alors compris, rendre accessible son atelier sous une forme dématérialisée devient un acte inopérant et vain.

\section{BIBLIOGRAPHIE}

Bouchardon, Serge, 2008, "C'est le geste du lecteur qui révèle la matérialité », dans A. Saemmer et M. Maza, (dir.), E-formes, Écritures visuelles sur support numérique, Saint-Étienne, Publications de l'Université de Saint-Étienne.

Derrida, Jacques, 1995, Mal d'archive : une impression freudienne, Paris, Éditions Galilée.

Donegan, Cheryl, 2009, "Allen Ruppersberg par Cheryl Donegan », BOMB - Artists in Conversation, $\mathrm{n}^{\circ}$ 109, http://bombmagazine.org/article/3330/allen-ruppersberg, consulté le 14 juin 2018.

Enwezor, Okwui (dir.), 2008, Archive Fever. Uses of the Document in Contemporary Art, cat. exp., New York, International Center for Photography/Steidl.

Fabre, Luis Felipe, 2012, « Letter to Iñaki Bonillas. [Delineated Photographs 2006] », J. R. Plaza Archive. Iñaki Bonillas, Zurich, Christoph Keller Edition by JRP I Ringier.

Foster, Hal, 2004, « An Archival Impulse », October, $\mathrm{n}^{\circ}$ 110, p. 3-22.

Fourmentraux, Jean-Paul, 2008, « Images mises au net. Entre art, média et communication numériques ", Études Photographiques, nº 22, [En ligne], http://journals.openedition.org/ etudesphotographiques/1010, consulté le 14 juin 2018.

Godfrey, Mark, 2007, « The Artists as Historian », October, n 120, p. 140-172.

Kittler, Friedrich, [1993] 2015, Mode protégé, Dijon, Presses du réel.

Krauss, Rosalind, printemps 1977, « Notes on the Index: Seventies Art in America », October, $\mathrm{n}^{\circ}$ 3, p. 68-81.

Laurensen, Pip, 2014, « Old Media, New Media? Significant Difference and the Conservation of Software-Based Art », New Collecting: Exhibiting and Audiences After New Media Art, Farnham, Ashagate, p. 77-82. 
Rehberg, Vivian, 2010, « Mapping the History of the Present ", Jeu de Paume Le Magazine, [En ligne], http://lemagazine.jeudepaume.org/2010/12/mapping-the-history-of-the-present-byvivian-rehberg/, consulté le 14 juin 2018.

Roelstrate, Dieter, 2009, « After The Historiographical Turn: Current Findings », e-flux, n ${ }^{\circ}$ 6, [En ligne], http://www.e-flux.com/journal/06/61402/after-the-historiographic-turn-currentfindings/, consulté le 14 juin 2018.

Ruppersberg, Allen, 2003, The New Five Foot Shelf, Bruxelles, éditions Micheline Szwajcer et Michèle Didier, Ljubljana, l'International Center of Graphic Art à Ljubljana, édition non paginée.

Schneller, Katia, 2007, « Sur les traces de Rosalind Krauss. La réception française de la notion d'index, 1977-1990 », Études photographiques, n² 21, [En ligne], http://journals.openedition.org/ etudesphotographiques/2483, consulté le 14 juin 2018.

Van Lier, Henri, 1982, « La Rhétorique des index », Cahiers de la photographie, n 5, p. 13-27.

Wall, Jeff, 1988, « Into the forest. Two sketches for studies of Rodney Graham's work », dans Rodney Graham: Works 1976-88, Vancouver, Vancouver Art Gallery, p. 9-37.

Zapperi, Giovanna (dir.), 2016, L'Avenir du passé. Art contemporain et politiques de l'archive, Rennes, PUR, coll. « Arts contemporains ».

\section{NOTES}

1. https://www.diaart.org/program/exhibitions-projects/type/web-project, consulté le 14 juin 2018.

2. Dès son lancement en 1974, la Dia Foundation a tout d'abord été précurseur dans l'exposition d'œuvres créées in situ. Dans cette continuité, les artist web project offrent aussi un accès immédiat à des œuvres et à leur environnement de création avec lequel elles se confondent.

3. Allen Ruppersberg, The New Five Foot Shelf, http://awp.diaart.org/ruppersberg/core.html, 2004.

4. Iñaki Bonillas, Words and Images, http://www.diaart.net/bonillas/index.html, 2014.

5. Entretien entre Iñaki Bonillas et Manuel Cirauqui, le 12 juin 2014 : «I found it enticing since the Internet is already a duplicate of the world. Because the frame was already a game of mirrors, I thought it could be profound to make another double by using words " (https://youtu.be/ AumF6vumSeg, consulté le 14 juin 2018).

6. Traduction française d'Emmanuel Guez d'une conférence donnée par le théoricien allemand en 1993. Les travaux de Friedrich Kittler, qui ont lancé l'archéologie des médias, suscitent actuellement un intérêt croissant dans le champ des recherches en art et en histoire de l'art.

7. Voir Pip Laurensen (2014: 77) : «It is central to the identity of software-based artworks that they are inherently transformative, that they do something in real time, something more than playback; so that the input is different from the output. Hence, it makes sense to ask whether these artworks analyze data or create visualizations. Whether they retrieve or select information, whether they sense presence, record events, produce or animate or translate content. »

8. Toute la difficulté du partage de l'expérience de ces projets tient à l'impossibilité de retrouver la configuration à laquelle on avait laissé les œuvres au moment de leur dernière activation. Afin de comprendre tous les enjeux analysés ici, l'activation des œuvres est indispensable.

9. «Bonillas is digitizing the archive, while simultaneously creating an extensive index of associated words that "mirrors" the image database. The relation between these two levels unfolds as the visitor explores the possibilities of matching text and image. Starting with a set of about 400 core images, the work will progressively expand until the entire J. R. Plaza Archive 
(approximately 3,800 images) is mapped out.» (http://awp.diaart.org/bonillas/, consulté le 14 juin 2018).

10. Ibid. : "In one photograph, for example, a woman is crying; the impulse might have been to tag a word that describes her abandonment, but that's maybe incorrect. That person may have been crying not out of sadness but out of joy. A plurality of meanings exist and could be derived from one's own personal archive of words, memory, or unique family history that is different from mine. »

11. L'apposition de cet adjectif «postérieur » aux utilisateurs indique qu'ils n'ont pas participé à l'indexation.

12. Une photographie de jeune femme en maillot de bain est ainsi indexée avec «narcissisme ».

13. Voir l'entretien avec Iñaki Bonillas, paru en ligne dans Artforum, le 18 novembre 2014. Lien de consultation permanent: http://artforum.com/words/id=49258. Consulté le 14 juin 2018 : «In others, a word as obvious as narcissism for a self-portrait may not have been initially tagged. My grandfather appears as a vain man in many of these photos; he thought of himself as a Hollywood actor and took on the persona of John Wayne as a cowboy with a gun in many. But no one on the project indexed "narcissism" for these photos. That word does exist on the website, though, because at least one user searched for it. All absent but sought-for tags are automatically added to a list at the bottom of the screen. More and more, I see these words and wonder why the person chose them. What are they seeing that I'm not? Sometimes the thing that you have in front of your eyes is impossible for you to see. That's the most extraordinary result of this project: our limitations. As Borges used to say, one can never finish describing an image for there is a whole ocean of words. "

14. Voir à ce sujet la synthèse de Katia Schneller (2007).

15. Voir Luis Felipe Fabre (2012 : 56) : «I can't get the thought out of my head that the images in your grandfather's photographs disappear at the same time as they turn into photographed images. » Luis Felipe Fabre s'appuie alors sur une série extraite de Delineated Photographs (2006) où l'artiste mexicain réinterprète le couvrement récurent par son grand-père du visage de son beaufrère Martín-Lunas, à l'aide d'un feutre noir. Sur ces photographies déjà mutilées, il marque d'un contour au crayon l'ensemble des silhouettes des figures photographiées, en recouvrant de noir l'espace entourant les figures. Il s'inspire alors du résultat formel d'une technique photographique - le dessin photogénique - utilisé par William Talbot en Angleterre à partir de 1834, et dont résulte une image négative développée sur le papier, obtenue par un noircissement direct à la lumière. Ne subsiste alors, dans un rendu très graphique, que le contraste entre l'absence des formes, marquée par leur silhouette blanche, et le fond totalement obscurci. L'empreinte apparaît de nouveau comme étant une donnée fondamentale dans le travail d'Iñaki Bonillas. Entre l'absence de l'image et l'apparition d'une nouvelle, la limite est instable, comme le démontre Luis Felipe Fabre, et cette donnée rend impossible, pour l'écrivain, la possibilité d'offrir un discours dont la pertinence puisse se maintenir dans la durée.

16. Voir aussi page 20 : «Au contraire, les empreintes-indices, en raison de leurs flottements et chevauchements, de leur immersion dans le bruit, sont saisies par déclenchements successifs et disparates. Dans telle portion de photo où il n'y avait d'abord que des plages sans imprégnant repérable, brusquement apparaît un événement, une structure, un objet qui viennent s'adjoindre à d'autres, ou bien les estompent, les déplacent, les incluent dans des ensembles différents. La rhétorique des index, quand elle intervient, canalise ces sautes brusques, mais sans les supprimer. »

17. Voir Allen Ruppersberg (2003 : n. p.). Traduction par l'auteure.

18. "Graham's object is a literary "thing" which is not really a book but which must appear as a book. The "thing" that appears as such is a pure manifestation of repetition-compulsion, pure in the sense that it is made visible not through an act of literary creation but through the organized ruination of literature. This ruination is effected not by writing a book but by binding one. » 
19. L'artiste américaine Martha Rosler se défend aussi avec malice de toute approche biographique, voir autobiographique, de la Martha Rosler Library, une bibliothèque au statut très ambivalent qui a circulé entre 2005 et 2009.

20. Voir l'entretien écrit et oral «Allen Ruppersberg par Cheryl Donegan ", BOMB - Artists in Conversation, $\mathrm{n}^{\circ}$ 109, [En ligne], http://bombmagazine.org/article/3330/allen-ruppersberg, consulté le 14 juin 2018.

\section{RÉSUMÉS}

Quel serait le sens des mots quand s'opèrent des tours de passe-passe entre l'indexation du réel par la photographie, l'indexation de la photographie par les mots et enfin l'indexation d'une création littéraire par des photographies? Les deux artist web projects d'Allen Ruppersberg et Iñaki Bonillas amènent à ce questionnement sous couvert d'un accès aux archives privées qu'ils détiennent.

What would be the meaning of words when sleights of hand take place between the indexation of reality by photographing, the indexation of photographs by words making and the indexation of literary works by photographs? The two artist web projects produced by Allen Ruppersberg and Iñaki Bonillas raise such issues under the guise of access to their private archives.

\section{INDEX}

Mots-clés : chose littéraire, œuvre numérique, Internet, photographie, index, archives, Dia Foundation, Ruppersberg (Allen), Bonillas (Iñaki), participatif

Keywords : literary thing, artist web project, internet photography, index, archives, Dia Foundation, Ruppersberg (Allen), Bonillas (Iñaki), participative

\section{AUTEUR}

\section{CONSTANCE MORÉTEAU}

Fondation AMIDEX - Aix Marseille Université 